\title{
A NEW MEASURE OF LINEAR LOCAL DEPENDENCE
}

\author{
ISMIHAN BAIRAMOV ${ }^{\mathrm{a}}$, SAMUEL KOTZ ${ }^{\mathrm{b}}$ and TOMASZ J. KOZUBOWSKI ${ }^{\mathrm{c}, *}$ \\ ${ }^{a}$ Izmir University of Economics, Department of Mathematics, Sakarya Caddesi 156, 35330 Izmir, \\ Turkey; ${ }^{\mathrm{b}}$ Dept. of Engineering Management and Systems Engineering, School of Engineering and \\ Applied Science, George Washington University, 1776/G Street, N.W., Washington, DC 20052, USA; \\ ${ }^{\mathrm{C}}$ University of Nevada, Dept. of Mathematics/084, Reno, NV 89557-0045, USA
}

(Received 20 November 2000; Revised 1 January 2002; In final form 6 May 2002)

\begin{abstract}
A new local dependence function based on regression concepts is introduced. This function can characterize the dependence structure of two random variables localized at the fixed point. Some properties of the local dependence function are given. Examples of important bivariate distributions are provided.
\end{abstract}

Keywords: Association; Bivariate distribution; Correlation; Dependence

\section{INTRODUCTION}

In recent years several important statistical papers have appeared, extending scalar association measure to local association functions. Bjerve and Doksum (1993), Doksum et al. (1994) and Blyth $(1993 ; 1994 a, b)$ introduce and discuss a "correlation curve", which is a generalization of the Pearson correlation coefficient. The correlation curve of Bjerve and Doksum (1993) is a local measure of the strength of association between random variables $X$ and $Y$, and is defined as

$$
\rho(x)=\frac{\sigma_{1} \beta(x)}{\left[\left(\left\{\sigma_{1} \beta(x)\right\}\right)^{2}+\sigma^{2}(x)\right]^{1 / 2}},
$$

where $\beta(x)=\mu^{\prime}(x)$ is the slope of the nonparametric regression $\mu(x)=E(Y \mid X=x)$, $\sigma^{2}(x)=\operatorname{Var}(Y \mid X=x)$ is the nonparametric residual variance, and $\sigma_{1}^{2}=\operatorname{Var}(X)$. The idea behind the construction of $\rho(x)$ is based on the fact that in the bivariate normal case

$$
\rho(x)=\rho=\frac{\sigma_{1} \beta}{\left[\left\{\sigma_{1} \beta\right\}^{2}+\sigma^{2}\right]^{1 / 2}},
$$

where $\beta$ is the slope of the regression line. Note that the measure $\rho(x)$ is not symmetric in $X$ and $Y$, and applies only when $X$ is a continuous random variable.

Jones (1996) provides a motivation for a local dependence function, the mixed partial derivative of the log density, proposed by Holland and Wang (1987). There are many ways of measuring dependence between two random variables. In a recent book, Nelsen

* Corresponding author. 
(1999) discusses various measures of dependencies, regarding "correlation coefficient" as a measure of the linear dependence between random variables, and using the term "measure of association" for measures such as Kendall's tau and Spearman's rho. Various measures of concordance and their properties are also described in Nelsen's book, providing relationships between measures of association and dependence of random variables.

This paper provides a description for a new local dependence function based on regression concepts. The measure is symmetric in $X$ and $Y$ and its expected value is approximately equal to the Pearson correlation coefficient. We define this new measure in Section 2, where we also discuss its basic properties. In Section 3 we provide examples of several important bivariate distributions. Graphs and tables are collected in Section 4.

\section{A LOCAL DEPENDENCE FUNCTION}

Let $X$ and $Y$ be random variables (r.v.'s) with marginal distribution functions (d.f.'s) and densities (p.d.f.'s) $F_{X}, f_{X}$ and $F_{Y}, f_{Y}$, respectively. Consider the following function of two variables

$$
H(x, y)=\frac{E\{(X-E(X \mid Y=y))(Y-E(Y \mid X=x))\}}{\left.\sqrt{E\left\{(X-E(X \mid Y=y))^{2}\right\}} \sqrt{E\left\{(Y-E(Y \mid X=x))^{2}\right.}\right\}},
$$

which is obtained from the expression of the Pearson correlation coefficient by replacing mathematical expectations $E X$ and $E Y$ by conditional expectations $E(X \mid Y=y)$ and $E(Y \mid X=x)$, respectively. By construction, $H(x, y)$ can be interpreted as a local dependence function characterizing the dependence between $X$ and $Y$ at the point $(x, y)$. After some simple algebra, (1) can be written as

$$
H(x, y)=\frac{\rho+\phi_{X}(y) \phi_{Y}(x)}{\sqrt{1+\phi_{X}^{2}(y)} \sqrt{1+\phi_{Y}^{2}(x)}},
$$

where

$$
\rho=\frac{\operatorname{Cov}(X, Y)}{\sigma_{X} \sigma_{Y}}
$$

is the Pearson correlation coefficient of $X$ and $Y$,

$$
\phi_{X}(y)=\frac{E X-E(X \mid Y=y)}{\sigma_{X}}, \quad \phi_{Y}(x)=\frac{E Y-E(Y \mid X=x)}{\sigma_{Y}},
$$

and $\sigma_{X}=\sqrt{\operatorname{Var}(X)}, \sigma_{Y}=\sqrt{\operatorname{Var}(Y)}$ are the standard deviations. The function $H(x, y)$ will be referred to as a local dependence function. The properties of $H(x, y)$ are given in the following lemma.

LEMMA 2.1 Let $(X, Y)$ have a bivariate distribution with finite second moments, Pearson correlation coefficient $\rho$, support $N_{X, Y}$, and local dependence function $H=H_{X, Y}$. Then,

$1^{\circ}$. If $X$ and $Y$ are independent, then $H(x, y)=0$ for all $(x, y) \in N_{X, Y}$.

$2^{\circ}$. $|H(x, y)| \leq 1$ for all $(x, y) \in N_{X, Y}$.

$3^{\circ}$. If $H(x, y)= \pm 1$ for some $(x, y) \in N_{X, Y}$, then $\rho \neq 0$.

$4^{\circ}$. If $Y=a X+b$, a.s., then $H(X, Y)=\operatorname{sign}(a)$, a.s.

$5^{\circ}$. If $\rho= \pm 1$ then $H(X, Y)= \pm 1$, a.s. 
$6^{\circ}$. If $\tilde{X}=a X+b$ and $\tilde{Y}=c Y+d$, then

$$
H_{\tilde{X}, \tilde{Y}}(\tilde{x}, \tilde{y})=\operatorname{sign}(a c) H_{X, Y}(x, y),
$$

where $\tilde{x}=a x+b$ and $\tilde{y}=c y+d$.

$7^{\circ}$. If $H(x, y)=0$ for all $(x, y) \in N_{X, Y}$, then either $E X=E(X \mid Y=y)$ or $E Y=E(Y \mid X=x)$ for all $(x, y) \in N_{X, Y}$, and $\rho=0$.

$8^{\circ}$. The point $\left(x^{*}, y^{*}\right)$ satisfying $\phi_{X}\left(y^{*}\right)=\phi_{Y}\left(x^{*}\right)=0$ is a saddle point of $H$ and $H\left(x^{*}, y^{*}\right)=\rho$.

Proof For proving $1^{\circ}$, note that when $X$ and $Y$ are independent, then $\rho=0$ and conditional expectations of $X$ and $Y$ coincide with expectations of $X$ and $Y$, so that $\phi_{X}(y)=\phi_{Y}(x)=0$ for $(x, y) \in N_{X, Y}$. Consequently, by (2), $H(x, y)=0$ for $(x, y) \in N_{X, Y}$.

For proving $2^{\circ}$, use Schwarz inequality.

For proving $3^{\circ}$, note that the condition $|H(x, y)|=1$ produces

$$
\left|\rho+\phi_{X}(y) \phi_{Y}(x)\right|=\sqrt{1+\phi_{X}^{2}(y)} \sqrt{1+\phi_{Y}^{2}(x)} .
$$

Squaring both sides of (5) and simplifying leads to

$$
\rho^{2}+2 \rho \phi_{X}(y) \phi_{Y}(x)=1+\phi_{X}^{2}(y)+\phi_{Y}^{2}(x),
$$

which is impossible if $\rho=0$.

For proving $4^{\circ}$, define the set $A=\{(x, y): y=a x+b, x=X(\omega), y=Y(\omega)\}$, which by assumption has probability one, and note that the function $H$ takes the constant value of $\operatorname{sign}(a)$ on $A$. Indeed, let $(x, y) \in A$. Then, $y=a x+b$ and $x=(y-b) / a$, so that $E(Y \mid X=x)=a x+b$ and $E(X \mid Y=y)=(y-b) / a=(a x+b-b) / a=x$, which implies that

$$
\phi_{X}(y)=\frac{E X-x}{\sigma_{X}} \quad \text { and } \quad \phi_{Y}(x)=\frac{a(E X-x)}{|a| \sigma_{X}} .
$$

Finally, substituting the above along with $\rho=a /|a|$ into (2), we obtain the assertion.

For proving $5^{\circ}$, first note that the condition $\rho= \pm 1$ implies that the distribution is concentrated on a straight line, and then use $4^{\circ}$.

For proving $6^{\circ}$, apply (2) noting that $\phi_{\tilde{X}}(\tilde{y})=\operatorname{sign}(a) \phi_{X}(y)$ and $\phi_{\tilde{Y}}(\tilde{x})=\operatorname{sign}(c) \phi_{Y}(x)$, while the correlation of $\tilde{X}$ and $\tilde{Y}$ is the same as $\operatorname{sign}(a c)$ times the correlation of $X$ and $Y$.

For proving $7^{\circ}$, note that if $H(x, y)=0$, then the numerator of (2) is equal to zero, so that

$$
E X E Y+\rho \sigma_{X} \sigma_{Y}=E X A_{Y}(x)+E Y A_{X}(y)-A_{X}(y) A_{Y}(x)
$$

where $A_{Y}(x)=E(Y \mid X=x)$ and $A_{X}(y)=E(X \mid Y=y)$. Differentiating (7) twice with respect to $x$ and $y$ leads to

$$
\frac{\mathrm{d}}{\mathrm{d} x} A_{Y}(x) \frac{\mathrm{d}}{\mathrm{d} y} A_{X}(y)=0
$$

so that either $A_{Y}(x)$ or $A_{X}(y)$ is equal to a constant. Suppose that $A_{X}(y)=E(X \mid Y=y)=C$. Then, $C=E C=E(E(X \mid Y))=E X$, so that

$$
\phi_{X}(y)=E X-A_{X}(y)=E X-E X=0
$$

and consequently $\rho=0$. A similar conclusion follows if $A_{Y}(x)=C$. The result follows.

For proving $8^{\circ}$, write

$$
H(x, y)=h\left(\phi_{X}(y), \phi_{Y}(x)\right)
$$


where

$$
h(t, z)=\frac{\rho+t z}{\sqrt{1+t^{2}} \sqrt{1+z^{2}}} .
$$

Then, the partial derivatives of $h$ are

$$
h_{t}(t, z)=\frac{z-\rho t}{\sqrt{1+z^{2}}\left(1+t^{2}\right)^{3 / 2}} \quad \text { and } \quad h_{z}(t, z)=\frac{t-\rho z}{\sqrt{1+t^{2}}\left(1+z^{2}\right)^{3 / 2}} .
$$

We see that for $|\rho|<1$, the only critical point of $h$ is the origin. Further, differentiating $h$ twice with respect to $t$ and $z$ we obtain

$$
h_{t t}(t, z)=\frac{-3 t z-\rho\left(1-2 t^{2}\right)}{\sqrt{1+z^{2}}\left(1+t^{2}\right)^{5 / 2}} \quad \text { and } \quad h_{z z}(t, z)=\frac{-3 t z-\rho\left(1-2 z^{2}\right)}{\sqrt{1+t^{2}}\left(1+z^{2}\right)^{5 / 2}},
$$

while the mixed derivative is

$$
h_{t z}(t, z)=h_{z t}(t, z)=\frac{1+\rho t z}{\left(1+z^{2}\right)^{3 / 2}\left(1+t^{2}\right)^{3 / 2}} .
$$

Consequently, at the critical point $(0,0)$ we have

$$
h_{t t}(0,0) h_{z z}(0,0)-\left[h_{t z}(0,0)\right]^{2}=\rho^{2}-1<0,
$$

showing that the origin is a saddle point of $h$. The result follows.

Remark Formula (2) suggests a possible estimator for the local dependence function $H(x, y)$. Nadaraya (1964) and Watson (1964) independently proposed the following estimate for the regression functions $E(X \mid Y=y)$ and $E(Y \mid X=x)$, respectively,

$$
A_{X}^{(n)}(y)=\frac{\sum_{i=1}^{n} X_{i} K\left(\left(y-Y_{i}\right) / h_{n}\right)}{\sum_{i=1}^{n} K\left(\left(y-Y_{i}\right) / h_{n}\right)} \quad \text { and } \quad A_{Y}^{(n)}(x)=\frac{\sum_{i=1}^{n} Y_{i} K\left(\left(x-X_{i}\right) / h_{n}\right)}{\sum_{i=1}^{n} K\left(\left(x-X_{i}\right) / h_{n}\right)}
$$

where $\left(X_{i}, Y_{i}\right), i=1,2, \ldots, n$ are the data, $K$ is an integrable kernel function with short tails, and $h_{n}$ is a width sequence tending to zero at an appropriate rate. Therefore we have the following estimate for $H(x, y)$ :

$$
\hat{H}_{n}(x, y)=\frac{\hat{\rho}_{n}+\left(\bar{X}-A_{X}^{(n)}(y)\right)\left(\bar{Y}-A_{Y}^{(n)}(x)\right) /\left(S_{X} S_{Y}\right)}{\sqrt{1+\left(\bar{X}-A_{X}^{(n)}(y)\right) / S_{X}^{2}} \sqrt{1+\left(\bar{Y}-A_{Y}^{(n)}(x)\right) / S_{Y}^{2}}},
$$

where

$$
\hat{\rho}_{n}=\frac{n \sum X_{i} Y_{i}-\sum_{i} X_{i} \sum_{j} Y_{j}}{\sqrt{n \sum_{i} X_{i}^{2}-\left(\sum_{i} X_{i}\right)^{2}} \sqrt{n \sum_{i} Y_{i}^{2}-\left(\sum_{i} Y_{i}\right)^{2}}}
$$

is a standard estimate for the Pearson correlation coefficient $\rho$ and $\bar{X}=1 / n \sum_{i} X_{i}$, $\bar{Y}=1 / n \sum_{i} Y_{i}, S_{X}^{2}=1 /(n-1) \sum_{i}\left(X_{i}-\bar{X}\right)^{2}$ and $S_{Y}^{2}=1 /(n-1) \sum_{i}\left(Y_{i}-\bar{Y}\right)^{2}$.

Remark The expected value of $H$ is obtained by weighted integration of $H$ with respect to the joint density $f$ of $(X, Y)$,

$$
E H=E[H(X, Y)]=\iint H(x, y) f(x, y) \mathrm{d} x \mathrm{~d} y
$$


and is always finite since $|H(x, y)| \leq 1$. As we see in the next section, this average nearly coincides with the Pearson correlation coefficient. Since $H(x, y)$ is basically a correlation when we concentrate at a particular point $(x, y)$, it is plausible that, in some smooth cases not far from linearity, the operation of averaging brings us back to the initial quantity - the classical correlation coefficient.

\section{EXAMPLES}

In this section we illustrate the concept of local linear dependence function by means of four examples, chosen to demonstrate the special features of the function at hand. For brevity, we shall skip most derivations and refer the reader to Bairamov et al. (2000) for a more detailed discussion.

\subsection{Bivariate Normal Distribution}

For a mean zero bivariate normal distribution with unit variances and correlation $\rho$, we have

$$
H(x, y)=\frac{\rho+\rho^{2} x y}{\sqrt{1+\rho^{2} y^{2}} \sqrt{1+\rho^{2} x^{2}}} .
$$

The Pearson correlation coefficient corresponds to the local dependence function at the origin. Figure 1 contains selected graphs of the local dependence function for various values of $\rho$. We see that $H(x, y)$ takes large values when $(x, y)$ lies near the diagonal $x=y$, and small values when $(x, y)$ lies in reverse sides. As shown in Bairamov et al. (2000), on any circle centered around the origin of fixed radius $r>0$, the function $H$ attains maximum value at $\theta=\pi / 4,5 \pi / 4$, and its minimum value at $\theta=3 \pi / 4,7 \pi / 4$ (in polar coordinates $x=r \cos \theta$ and $y=r \sin \theta)$. Moreover, for any fixed $\theta \in[0,2 \pi)$, the function $h(r)=H(r \cos \theta, r \sin \theta)$ admits the following limit at infinity:

$$
\lim _{r \rightarrow \infty} h(r)=\left\{\begin{aligned}
-1, & \text { for } \theta \in(\pi / 2, \pi) \cup(3 \pi / 2,2 \pi), \\
0, & \text { for } \theta=0, \pi / 2, \pi, 3 \pi / 2 \\
1, & \text { for } \theta \in(0, \pi / 2) \cup(\pi, 3 \pi / 2) .
\end{aligned}\right.
$$

Thus, we may have a point $(x, y)$ for which the density $f$ is almost zero, and yet the local dependence function $H$ is close to its maximal value of one. Bairamov et al. (2000) compared values of $H(x, y)$ and $f(x, y)$ for various choices of $x$ and $y$, finding that when $(x, y)$ is near the origin (where the density attains the largest value), the values of $H$ concentrate tightly near $\rho$, while the values of $H$ become more spread out and eventually cover almost the entire range from -1 to 1 as the point $(x, y)$ gets further away from the origin (and values of $f$ decrease towards zero).

The average value $E H$ given by (16) can be approximated through the numerical integration of the function $H(x, y) f(x, y)$. Table I contains selected numerical values of $\rho$ and $E H=E H(X, Y)$. We used Monte Carlo integration with a sample size $n=10,000$ to evaluate the values of $E H$. Remarkably, $\rho$ and $E H$ are in close agreement, especially for values of $\rho$ near zero.

\subsection{Farlie-Gumbel-Morgenstern Distribution}

Consider the one-parameter family of Farlie-Gumbel-Morgenstern (FGM) distributions with uniform marginals, given by the p.d.f.

$$
f_{\alpha}(x, y)=1+\alpha(1-2 x)(1-2 y), \quad 0 \leq x, y \leq 1, \quad-1 \leq \alpha \leq 1 .
$$


For generalizations and further discussion, see Johnson and Kotz $(1975 ; 1977)$. Here, the local dependence function takes the form

$$
H(x, y)=\frac{\rho+3 \rho^{2}(1-2 x)(1-2 y)}{\sqrt{1+3 \rho^{2}(1-2 x)^{2}} \sqrt{1+3 \rho^{2}(1-2 y)^{2}}},
$$

where $\rho=\alpha / 3$ is the Pearson correlation coefficient. The local dependence function coincides with the correlation coefficient at the point of symmetry $(x, y)=(1 / 2,1 / 2)$. In Figure 2 we present selected graphs of local dependence function for FGM distributions. Unlike the normal case, where the value of $H$ may approach one even though the density approaches zero, here the dependence function is close to zero when the density is close to zero, and the dependence gets stronger as the values of $f_{\alpha}$ increase.

In Table II we provide some numerical values of $E H$ for selected values of $\alpha$. Again, the average values of $H$ are remarkably close to the Pearson correlation coefficient. In fact, as shown in Bairamov et al. (2000), we have

$$
E H=E H(\alpha)=E H(X, Y)=I_{1}(\alpha)+I_{2}(\alpha),
$$

where

$$
\begin{aligned}
& R(x)=\frac{|x|}{\sqrt{3}}+\sqrt{\frac{x^{2}}{3}+1}, \\
& I_{1}(x)=\frac{1}{x} \log ^{2} R(x)=\frac{1}{3} x-\frac{1}{27} x^{3}+\frac{8}{1215} x^{5}+O\left(x^{7}\right) \quad(x \rightarrow 0), \\
& I_{2}(x)=\frac{1}{4 x}\left(\sqrt{3+x^{2}}-\frac{3}{|x|} \log R(x)\right)^{2}=\frac{1}{27} x^{3}-\frac{1}{135} x^{5}+O\left(x^{7}\right) \quad(x \rightarrow 0) .
\end{aligned}
$$

Thus, for $\alpha$ close to zero, we have $E H(\alpha)=\rho+O\left(\alpha^{5}\right)$.

\subsection{Bivariate Exponential Conditionals Distribution}

Consider the following bivariate distribution, referred to as a bivariate exponential conditionals (BEC) distribution by Arnold and Strauss (1988), with joint p.d.f.

$$
f(x, y)=k \exp (-x-y-\delta x y), \quad 0 \leq x, y,<\infty, \quad \delta \geq 0,
$$

where

$$
k=\frac{\delta \exp (-1 / \delta)}{E_{1}(1 / \delta)}
$$

and

$$
E_{1}(z)=\int_{z}^{\infty} \frac{e^{-t}}{t} \mathrm{~d} t
$$

is the exponential integral function (see, e.g., Abramowitz and Stegun, 1965, formula 5.1.1). Here the conditional distributions are exponential and the marginal distributions are independent for $\delta=0(k=1)$. For this family, the local dependence function is

$$
H(x, y)=\frac{\delta+k-k^{2}+(k-1-\delta /(1+\delta y))(k-1-\delta /(1+\delta x))}{\sqrt{k \delta+k-k^{2}+(k-1-\delta /(1+\delta x))^{2}} \sqrt{k \delta+k-k^{2}+(k-1-\delta /(1+\delta y))^{2}}},
$$


and we have

$$
H\left(\frac{1}{k-1}-\frac{1}{\delta}, \frac{1}{k-1}-\frac{1}{\delta}\right)=\frac{k-k^{2}+\delta}{k \delta+k-k^{2}}=\rho .
$$

In Figure 3 we provide selected graphs of the local dependence function. The expected value of $H$ is finite, and can be approximated through the numerical integration of the function $H(x, y) f(x, y)$ for any given value of $\delta$. Table III contains numerical values of $\rho$ and $E H=E H(X, Y)$ for selected values of $\delta$. We used Monte Carlo integration with sample size $n=10,000$ to evaluate the values of $E H$. To simulate random samples from the BEC distribution we used the rejection algorithm described in Arnold and Straus (1988). It is apparent that $\rho$ and $E H$ are in close agreement, especially for small values of $\delta$.

\subsection{Gumbel's Bivariate Exponential Distribution}

In this section we consider the distribution of a random vector $(X, Y)$ with p.d.f.

$$
f(x, y)=\exp (-x-y-\delta x y)[(1+\delta x)(1+\delta y)-\delta], \quad x, y>0, \quad 0 \leq \delta \leq 1,
$$

which was studied in Gumbel (1960). As the marginal distributions of $X$ and $Y$ are standard exponential, we shall refer to the above distribution as Gumbel's bivariate exponential (GBE) distribution. The correlation of $X$ and $Y$ is

$$
\rho=\left(\frac{1}{\delta}\right) e^{1 / \delta} E_{1}\left(\frac{1}{\delta}\right)-1
$$

For $\delta=0$, the variables are independent with $\rho=0$. (At the other extreme, the correlation is about -0.4037 for $\delta=1$.) The local dependence function takes the form

$$
H(x, y)=\frac{\rho+\left(1-(1+\delta+\delta x) /(1+\delta x)^{2}\right)\left(1-(1+\delta+\delta y) /(1+\delta y)^{2}\right)}{\sqrt{1+\left(1-(1+\delta+\delta x) /(1+\delta x)^{2}\right)^{2}} \sqrt{1+\left(1-(1+\delta+\delta y) /(1+\delta y)^{2}\right)^{2}}}
$$

and coincides with $\rho$ when

$$
x=y=\frac{\sqrt{1+4 \delta}-1}{2 \delta} .
$$

Figure 4 contains plots of the local dependence function for selected GBE distributions. Compared with BEC distributions, one may notice that although densities of BEC and GBE may be quite different, the two distributions seem to have very similar local dependence structures.

Finally, we calculate the expected value of $H$ for selected values of $\delta$ and compare it with the correlation coefficient. In the calculation we numerically integrate the function $H(x, y) f(x, y)$ via Monte Carlo integration with sample size $n=10,000$. To generate variates from the GBE distribution, we followed the conditional distribution approach described in Johnson (1987, p. 197). ${ }^{1}$ Table IV contains values of $\rho$ and $E H=E H(X, Y)$ for selected values of $\delta$. Again, the two quantities are in close agreement.

\footnotetext{
${ }^{1}$ There seems to be a misprint in the algorithm presented in Johnson (1987); a corrected version can be found in Bairamov et al. (2000).
} 


\section{GRAPHS AND TABLES}
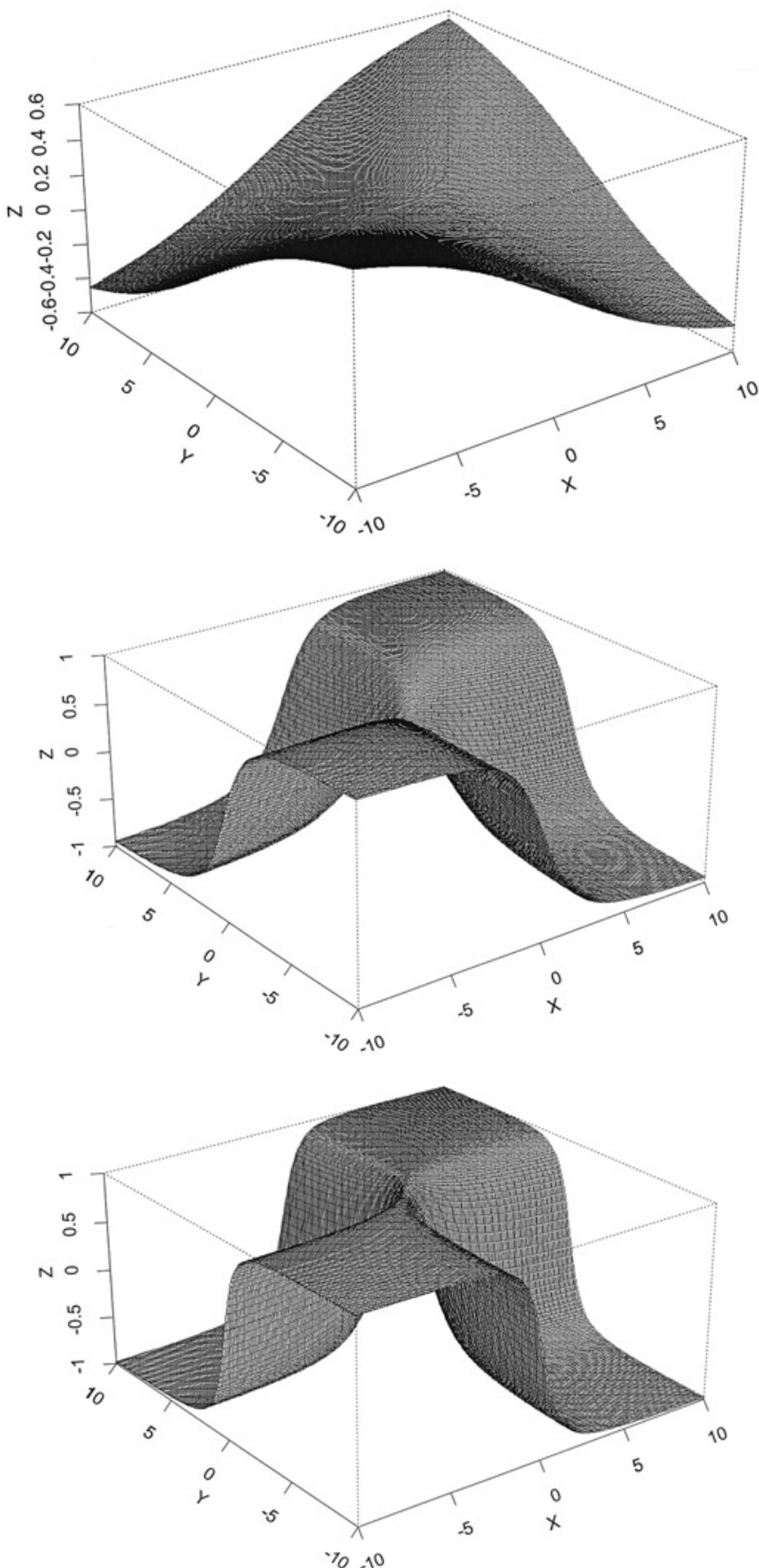

FIGURE 1 Perspective plots (left) and contour plots (right) of $H(x, y)$ for bivariate normal distribution with vector mean zero, unit variances, and correlation equal to 0.1 (top), 0.5 (middle), and 0.95 (bottom). 

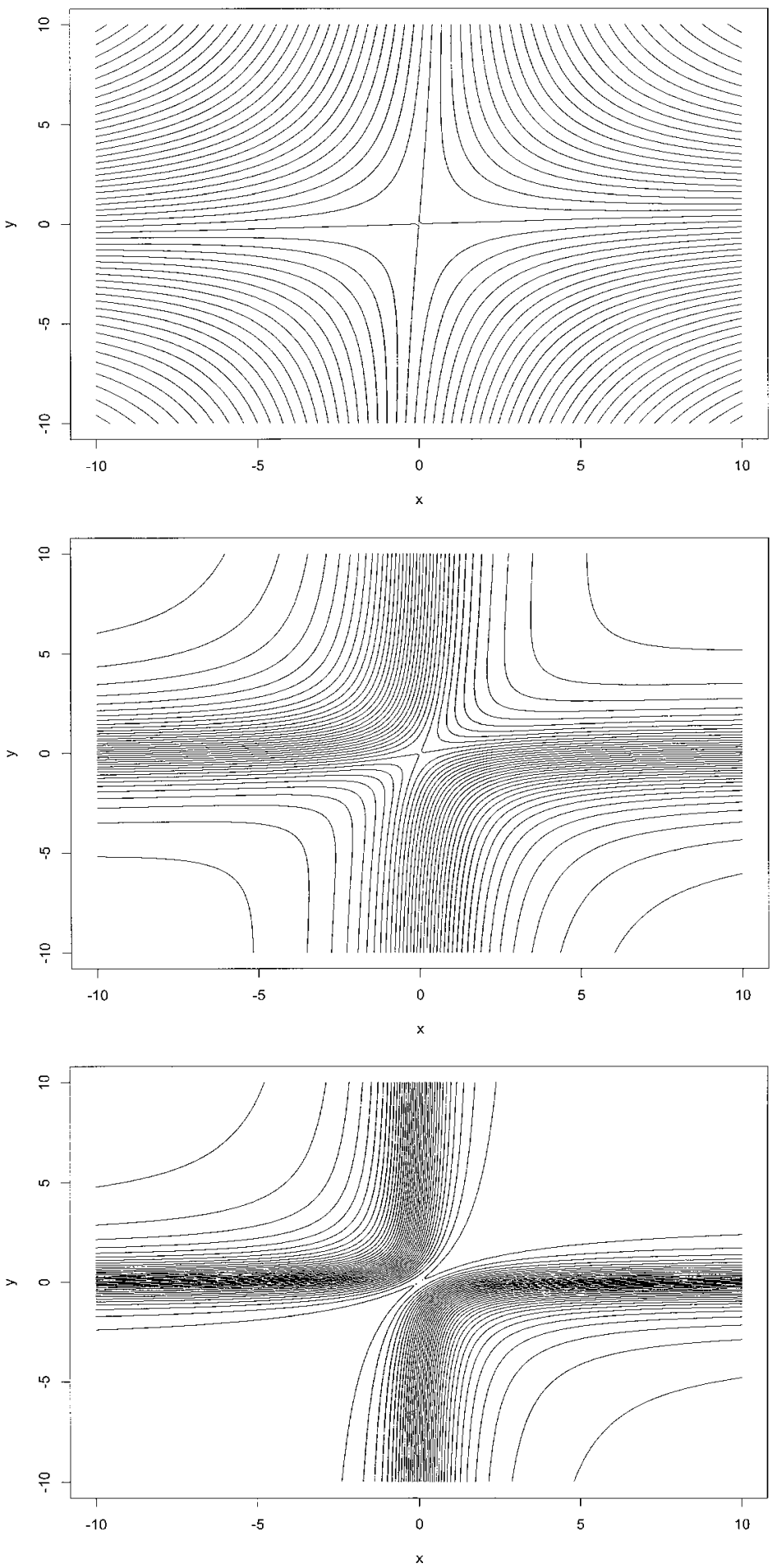

FIGURE 1 (continued). 

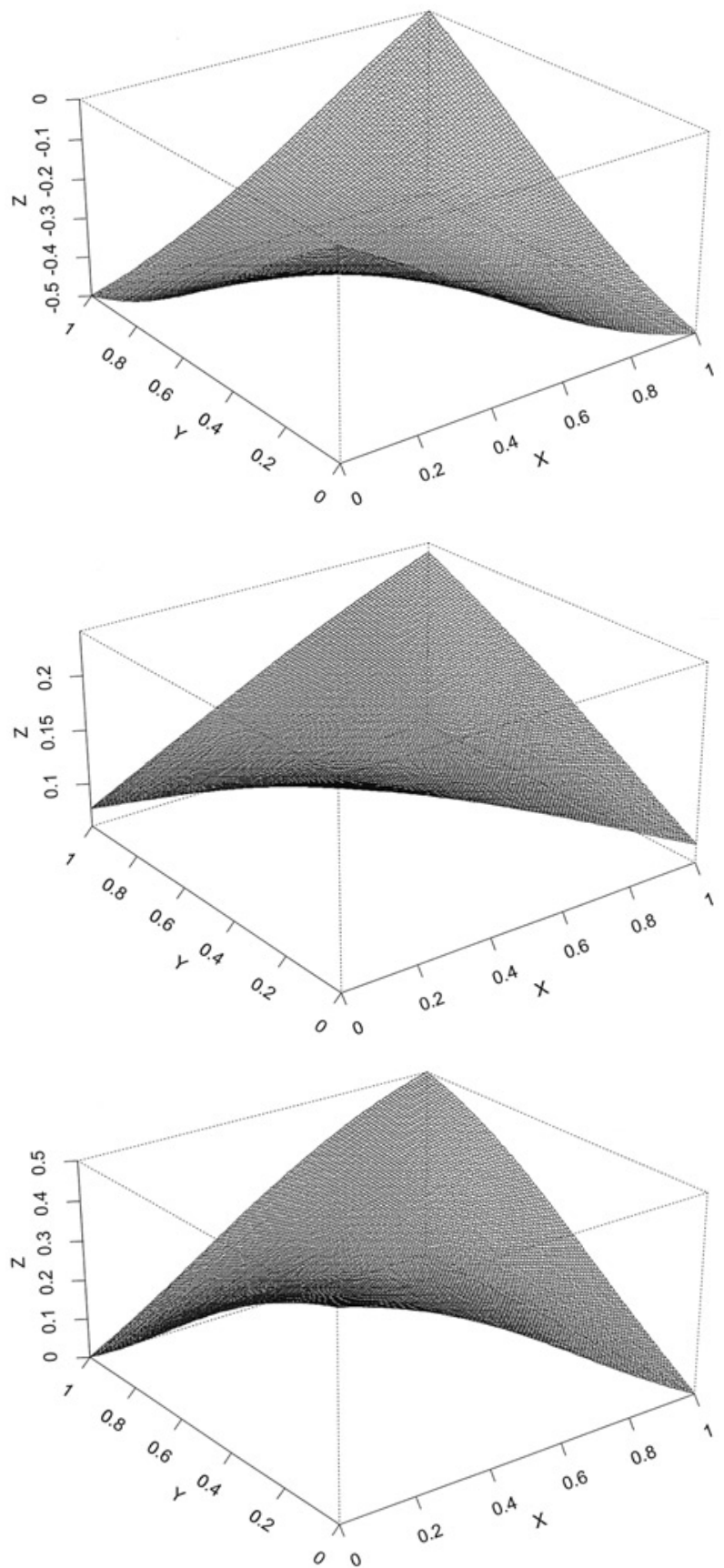

FIGURE 2 Perspective plots (left) and contour plots (right) of $H(x, y)$ for FGM distributions with uniform marginals and parameters $\alpha$ equal to -1 (top), 0.5 (middle), and 1 (bottom). 

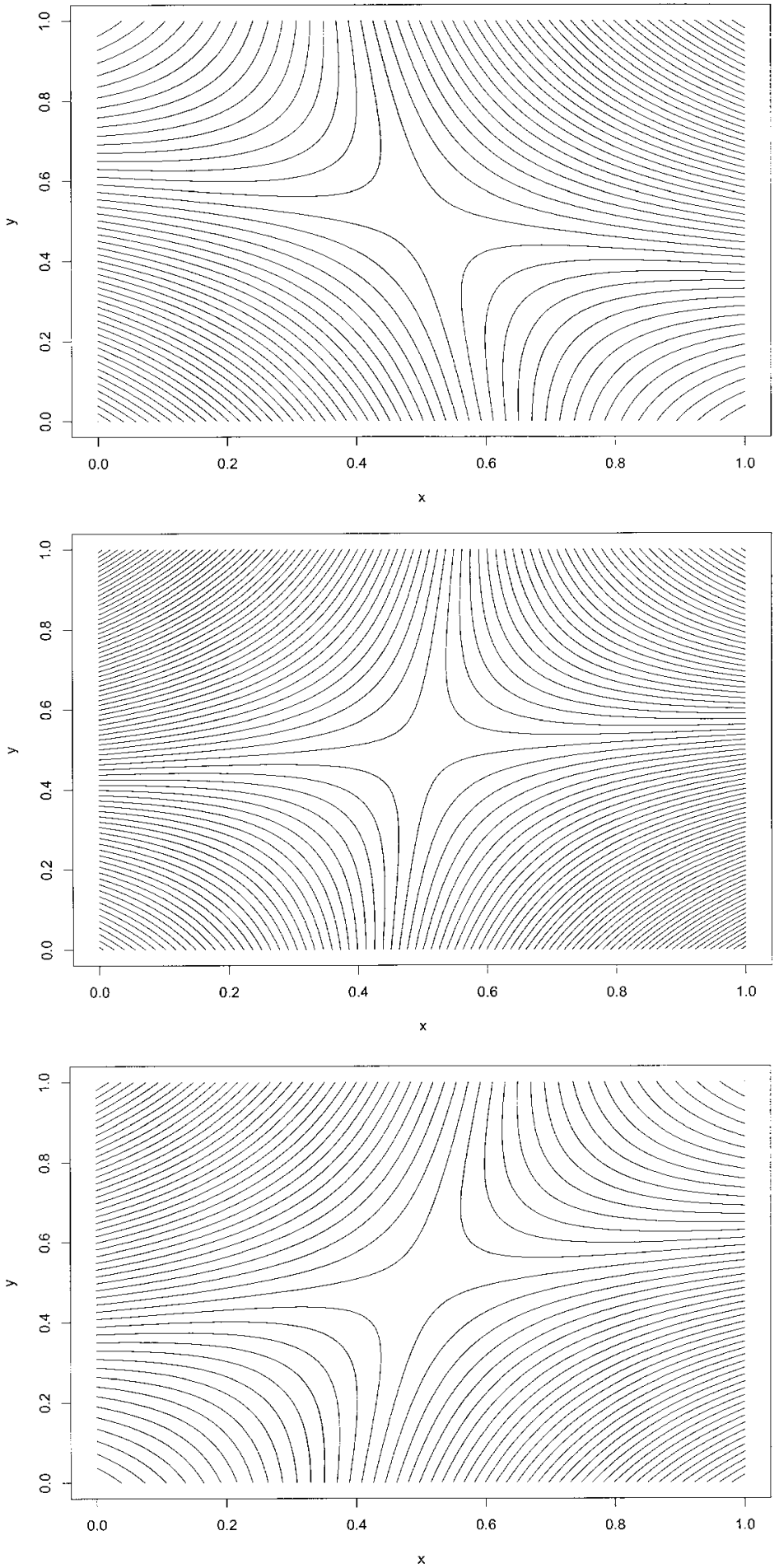

FIGURE 2 (continued). 

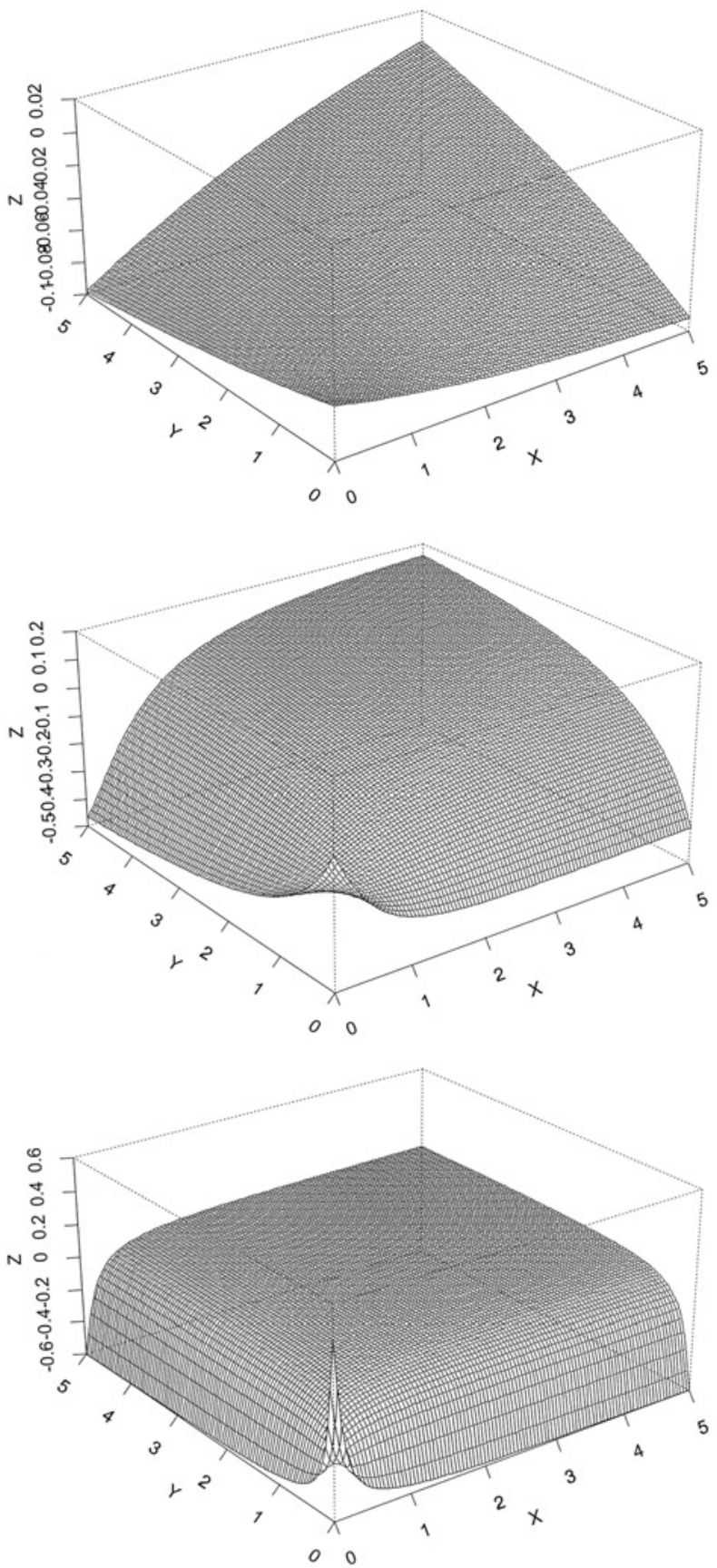

FIGURE 3 Perspective plots (left) and contour plots (right) of $H(x, y)$ for BEC distributions with parameter equal to 0.1 (top), 1 (middle), and 10 (bottom). 

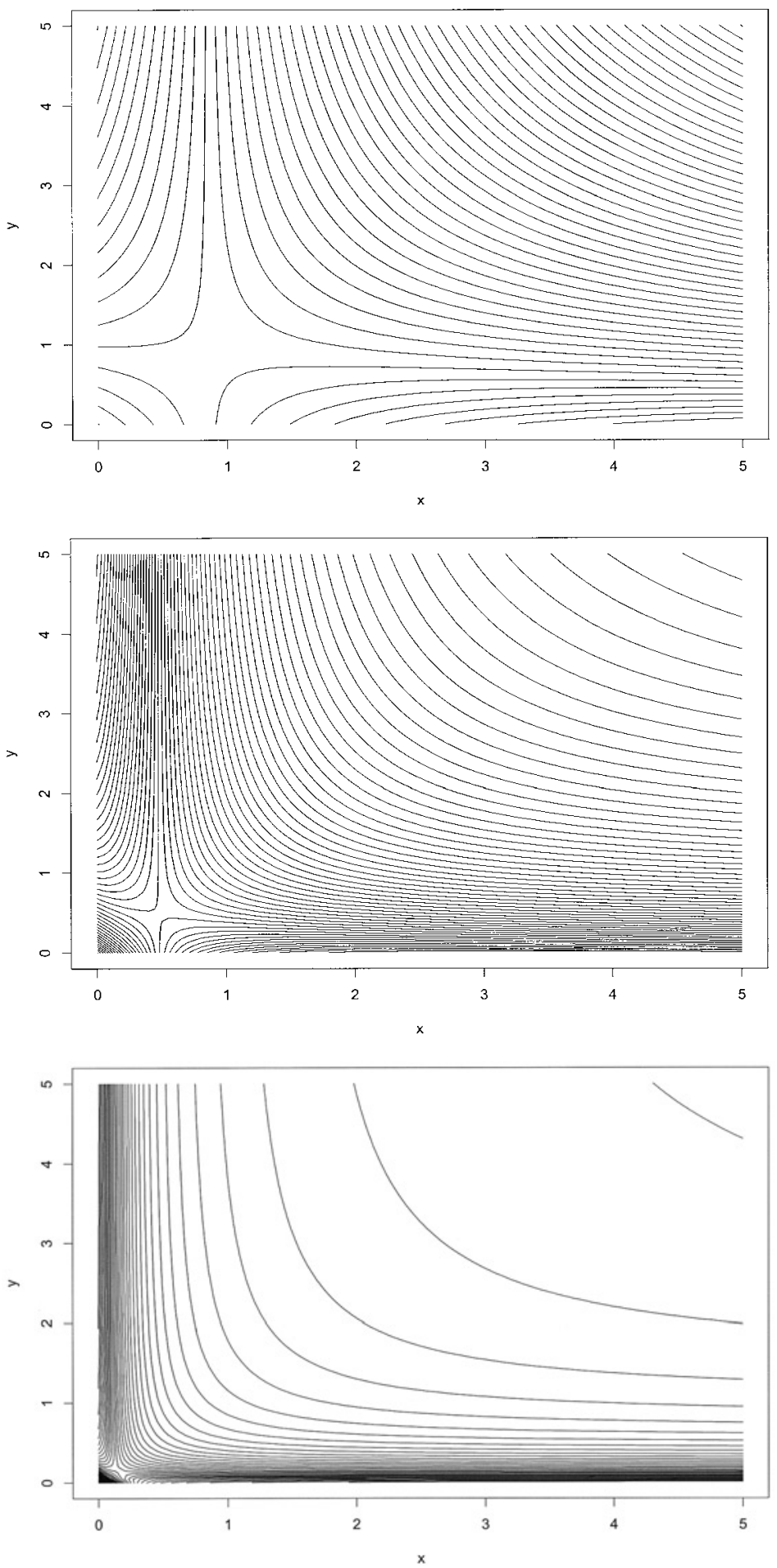

FIGURE 3 (continued). 

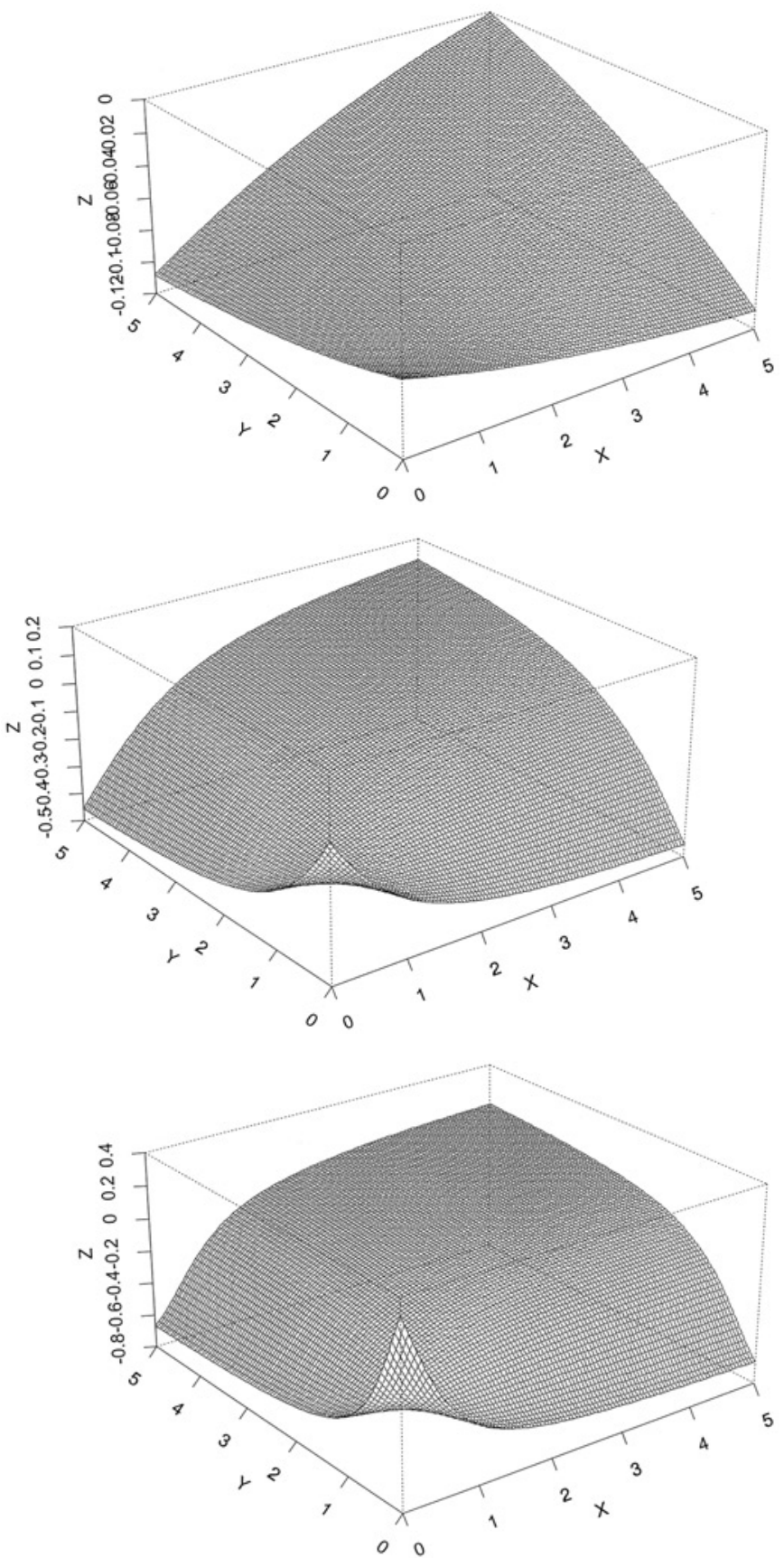

FIGURE 4 Perspective plots (left) and contour plots (right) of $H(x, y)$ for Gumbel's bivariate exponential distributions with parameter $\delta$ equal to 0.1 (top), 0.5 (middle), and 1 (bottom). 

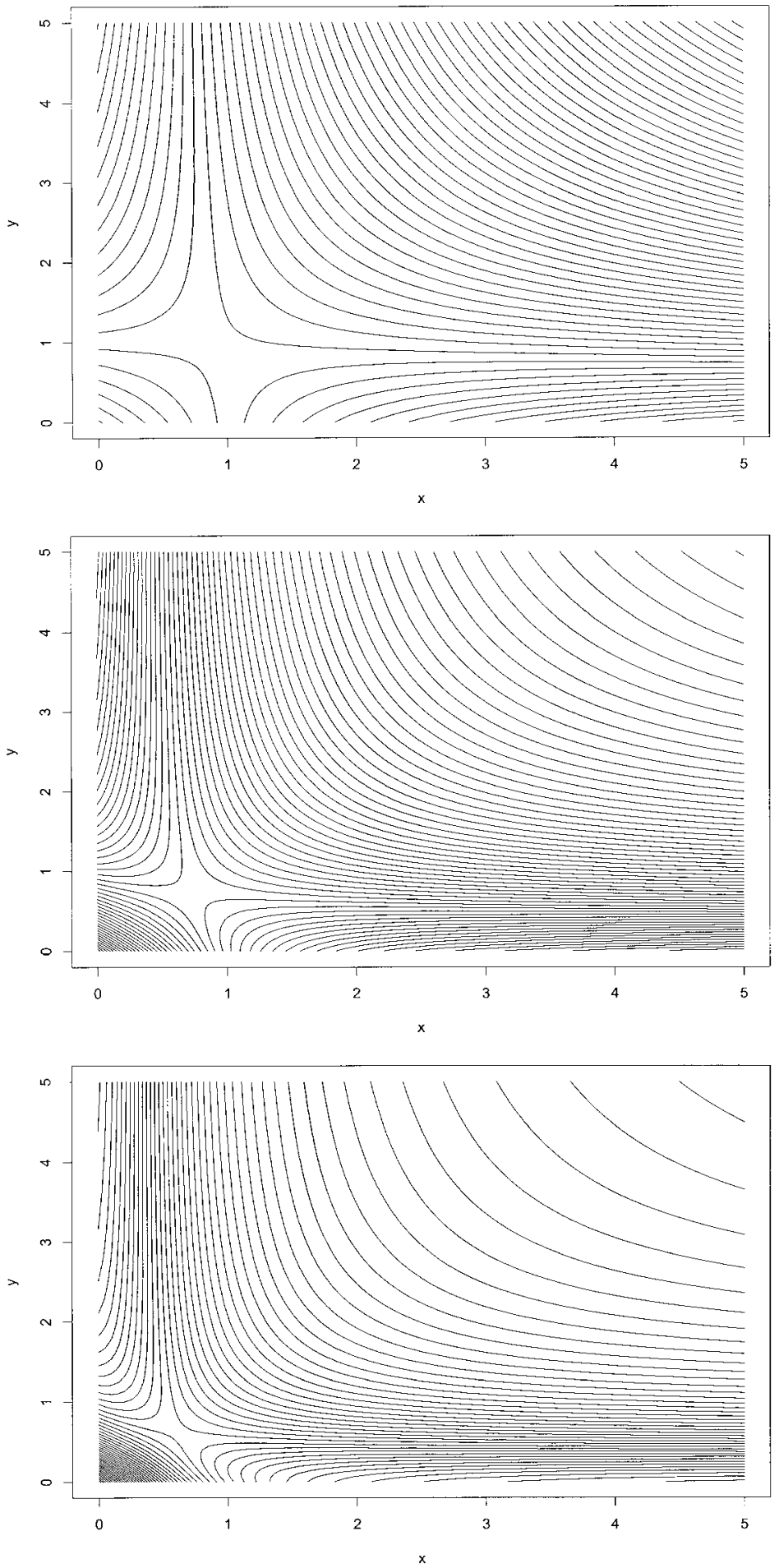

FIGURE 4 (continued). 
TABLE I Selected Numerical Values of $\rho$ and $E H=E H(X, Y)$ for Bivariate Normal Distributions with Mean Zero and Unit Variances.

\begin{tabular}{lccccccc}
\hline$\rho$ & 0.01000 & 0.02500 & 0.05000 & 0.10000 & 0.25000 & 0.50000 & 0.95000 \\
$E H$ & 0.010000 & 0.02500 & 0.05000 & 0.09992 & 0.24976 & 0.49385 & 0.94383 \\
\hline
\end{tabular}

TABLE II Selected Numerical Values of $E H(\alpha)$ for FGM Distribution with Uniform Marginals.

\begin{tabular}{lllllllll}
\hline$\alpha$ & 0.00000 & 0.02500 & 0.05000 & 0.10000 & 0.25000 & 0.50000 & 0.75000 & 1.00000 \\
$\rho=\alpha / 3$ & 0.00000 & 0.00833 & 0.01667 & 0.03333 & 0.08333 & 0.16667 & 0.25000 & 0.33333 \\
$E H(\alpha)$ & 0.00000 & 0.00833 & 0.01667 & 0.03333 & 0.08333 & 0.16664 & 0.24984 & 0.33273 \\
\hline
\end{tabular}

TABLE III Selected Numerical Values of $\rho$ and $E H=E H(X, Y)$ for BEC Distributions with Selected Values of $\delta$.

\begin{tabular}{|c|c|c|c|c|c|c|c|c|}
\hline$\delta$ & 0.025 & 0.05 & 0.1 & 0.25 & 0.5 & 1 & 2.5 & 5 \\
\hline$\rho$ & -0.0228 & -0.0421 & -0.0734 & -0.1356 & -0.1932 & -0.2492 & -0.3034 & -0.3224 \\
\hline$E H$ & -0.0228 & -0.0421 & -0.0734 & -0.1357 & -0.1940 & -0.2514 & -0.3128 & -0.3397 \\
\hline
\end{tabular}

TABLE IV Selected Numerical Values of $\rho$ and $E H=E H(X, Y)$ for Gumbel's Bivariate Exponential Distributions with Various Parameters $\delta$.

\begin{tabular}{|c|c|c|c|c|c|c|c|}
\hline$\delta$ & 0 & 0.025 & 0.05 & 0.1 & 0.25 & 0.5 & 1 \\
\hline$\rho$ & 0 & -0.02384 & -0.04563 & -0.08437 & -0.17462 & -0.27734 & -0.40365 \\
\hline$E H$ & 0 & -0.02385 & -0.04561 & -0.08435 & -0.17552 & -0.28076 & -0.41991 \\
\hline
\end{tabular}

\section{Acknowledgement}

We thank the anonymous referees for their useful comments.

\section{References}

Abramowitz, M. and Stegun, I. A. (1965). Handbook of Mathematical Functions. Dover Publications, New York. Arnold, B. C. and Strauss, D. (1988). Bivariate distributions with exponential conditionals. J. Amer. Statist. Assoc., 83(402), 522-527.

Bairamov, I., Kotz, S. and Kozubowski, T. J. (2000). A new measure of local dependence. Technical Report No. 362, Department of Statistics and Applied Probability, University of California at Santa Barbara.

Bjerve, S. and Doksum, K. (1993). Correlation curves: Measures of association as functions of covariate values. Ann. Statist., 21(2), 890-902.

Blyth, S. (1993). A note on correlation curves and Chernoff's inequality. Scand. J. Statist., 20(4), 375-377.

Blyth, S. (1994a). Karl Pearson and the correlation curve. Internat. Statist. Rev., 62(3), 393-403.

Blyth, S. (1994b). Measuring local association: an introduction to the correlation curve. Sociol. Meth., 24, $171-197$.

Doksum, K., Blyth, S., Bradlow, E., Meng, X. L. and Zhao, H. (1994). Correlation curves as local measures of variance explained by regression. J. Amer. Statist. Assoc., 89(426), 571-582.

Gumbel, E. J. (1960). Bivariate exponential distributions. J. Amer. Statist. Assoc., 55, 698-707.

Holland, P. W. and Wang, Y. J. (1987). Regional dependence for continuous bivariate densities. Comm. Statist. Theory Methods, 16(1), 193-206.

Johnson, M. E. (1987). Multivariate Statistical Simulation. Wiley, New York.

Johnson, N. L. and Kotz, S. (1975). On some generalized Farlie-Gumbel-Morgenstern distributions. Comm. Statist., 4, 415-427.

Johnson, N. L. and Kotz, S. (1977). On some generalized Farlie-Gumbel-Morgenstern distributions. II. Regression, correlation and further generalizations. Comm. Statist. Theory Methods, A6(6), 485-496.

Jones, M. C. (1996). The local dependence function. Biometrika, 83(4), 899-904.

Nadaraya, E. A. (1964). On estimating regression. Theory Probab. Appl., 9, 141-142.

Nelsen, R. B. (1999). An Introduction to Copulas, Lecture Notes in Statistics, Vol. 139. Springer-Verlag, New York.

Watson, G. S. (1964). Smooth regression analysis. Sankhya A, 26, 359-372. 